\title{
Creating and testing a survey to assess the impact of renewable energy technologies on quality of life
}

Ref: Environmental Health Reviews. 2013 56(4):103-111.

One author was inadvertently omitted at the time of publication. The correct list of authors is as follows.

Tanya Christidis*, Claire Paller ${ }^{\dagger}$, Shannon Majowicz ${ }^{\dagger}$, Phil Bigelow $^{\dagger}$, Ashley Wilson $^{\dagger}$, Sehar Jamal ${ }^{\dagger}$, and Susan Yates ${ }^{\dagger}$ *School of Planning, University of Waterloo, 200 University Avenue West, Waterloo, ON, N2L 3G1.

'School of Public Health and Health Systems, University of Waterloo, 200 University Avenue West, Waterloo, ON, N2L 3G1. 